\title{
Packing Polydisperse Colloids into Crystals: When Charge-Dispersity Matters
}

\author{
Guillaume Bareigts $\odot,{ }^{1}$ Pree-Cha Kiatkirakajorn $\odot,{ }^{2}$ Joaquim Li, ${ }^{3}$ Robert Botet $\odot,{ }^{4}$ Michael Sztucki, ${ }^{5}$ \\ Bernard Cabane $\oplus^{3}$ Lucas Goehring $\odot,{ }^{6, *}$ and Christophe Labbez $\oplus^{1, \uparrow}$ \\ ${ }^{1}$ ICB, CNRS UMR 6303, Université Bourgogne Franche-Comté, 21000 Dijon, France \\ ${ }^{2}$ Max Planck Institute for Dynamics and Self-Organisation (MPIDS), Göttingen 37077, Germany \\ ${ }^{3}$ LCMD, CNRS UMR 8231, ESPCI, 10 rue Vauquelin, 75231 Paris Cedex 05, France \\ ${ }^{4}$ Physique des Solides, CNRS UMR 8502, Université Paris-Saclay, F-91405 Orsay, France \\ ${ }^{5}$ ESRF-The European Synchrotron, CS40220, 38043 Grenoble Cedex 9, France \\ ${ }^{6}$ School of Science and Technology, Nottingham Trent University, Nottingham NG11 8NS, United Kingdom
}

(Received 24 September 2019; accepted 6 January 2020; published 5 February 2020)

\begin{abstract}
Monte Carlo simulations, fully constrained by experimental parameters, are found to agree well with a measured phase diagram of aqueous dispersions of nanoparticles with a moderate size polydispersity over a broad range of salt concentrations, $c_{s}$, and volume fractions, $\phi$. Upon increasing $\phi$, the colloids freeze first into coexisting compact solids then into a body centered cubic phase (bcc) before they melt into a glass forming liquid. The surprising stability of the bcc solid at high $\phi$ and $c_{s}$ is explained by the interaction (charge) polydispersity and vibrational entropy.
\end{abstract}

DOI: 10.1103/PhysRevLett.124.058003

How do polydisperse particles pack and order? This basic question concerns diverse systems, including granular beads, microemulsions, microgels, macromolecules, and solid nanoparticles and is, thus, largely debated. For a fluid of hard-sphere (HS) particles, Pusey et al. [1,2] proposed a critical value of polydispersity $(\delta)$, above which particles would not crystallize. This concept of a terminal polydispersity was first based on experimental observations, and later supported also by numerical simulations [3,4]. However, using simulations of HS systems, Kofke et al. [5] found that the concept of a terminal polydispersity should only apply to a solid phase, rather than the entire system of particles. More precisely, that a stable crystalline phase whose constituent components exceeded a polydispersity of $5.7 \%$ could not be formed from a fluid phase. Questioning the ultimate fate of an amorphous solid of high $\delta$, they proposed that fractionation should enable an HS fluid of arbitrary polydispersity to precipitate in a fcc solid phase in coexistence with a fluid phase. Sollich et al. $[6,7]$ further theorized that, when compressed, a relatively polydisperse HS system should crystallize into a myriad of coexisting fcc crystalline phases, each having a distinct size distribution and a narrower $\delta$ than the mother distribution, as in Fig. 1(a).

Our recent experiments [8] on dispersions of charged hard spheres (CS) with a broad and continuous size polydispersity $(\delta=14 \%)$ empirically demonstrated the case of the fractionation of a colloidal fluid into multiple coexisting phases. Interestingly, this crystallization turns out to be more complex than that theorized by Sollich et al. for HS particles. Indeed, as in Fig. 1(b), the CS were observed to coexist in a fluid phase, a bcc lattice and a
Laves $\mathrm{MgZn}_{2}$ superlattice. The latter had been previously known only from binary distributions of particles [10-12]. Matching lattice simulations can also reproduce the experimental findings, including the Laves phase $[8,13]$. Very recent simulations $[9,14,15]$ with polydisperse HS particles of $\delta>6 \%$ show a similar, or even greater, level of

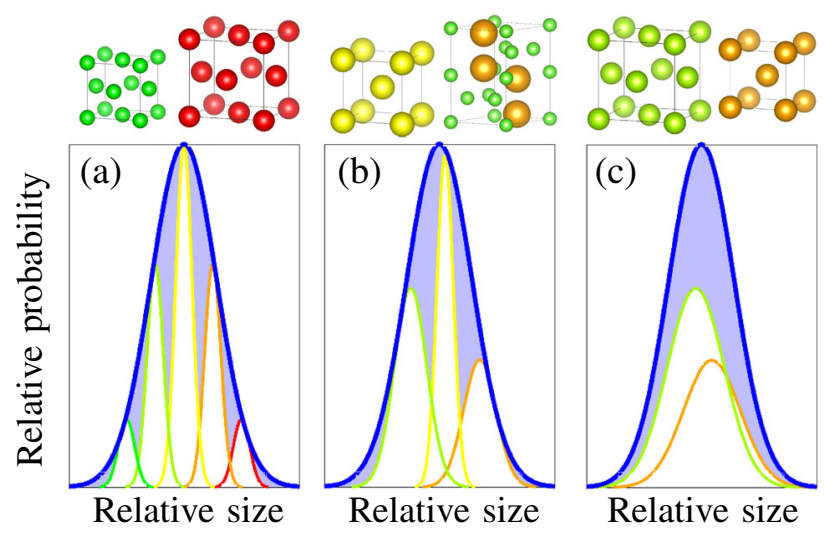

FIG. 1. Colloidal crystallization in a polydisperse system can lead to (a) a set of distinct crystals of the same structure (e.g., fcc) and narrow monomodal size distributions, which together span the available range of particle sizes [6]; (b) more complex phases such as $A B_{2}$ [8] or $A B_{13}$ [9] structures, which utilize a bimodal subset of particles. These may coexist with simpler phases (e.g., as above, bcc [8]); (c) The appearance of crystals of different structures (e.g., bcc, fcc, hcp) and monomodal size distributions, as reported in this Letter. In all sketches, the shaded area shows the parent particle size distribution while the various open curves describe the particles found in any specific crystal structure and site. 
complexity and thus indicate that our findings with CS are representative of a more general rule: polydispersity enables complex crystal formation. In particular, Frank-Kasper phases, as well as various Laves $A B_{2}$ and $A B_{13}$ phases were found in simulations of HS particles of $\delta$ from $6 \%$ to $24 \%$ and at high packing fractions $(\phi)$. These results are also in line with the earlier simulations of Fernandez et al. [16], of neutral soft spheres, even though the exact natures of the crystal phases obtained there were not identified. On the other hand, the coexistence of multiple crystal phases of the same symmetry, but different lattice constants, has only been observed in systems of platelike particles [17].

Here, we demonstrate that even with a moderate size polydispersity CS systems can show a complex phase behavior. This is achieved on a similar CS system to that in [8] but with a more moderate size dispersity (9\%). The magnitude and polydispersity of the charge, and thus of the interaction polydispersity, are tuned with the salt concentration, $c_{s}$, and the $p \mathrm{H}$ of the bulk solution (see the Supplemental Material [18]). Using x-ray scattering methods, the $c_{s}-\phi$ phase diagram is constructed. We observe that on gradually increasing the osmotic compression the CS fluid crystallizes and fractionates into coexisting phases of different structures, i.e., bcc, fcc, and hcp, as in Fig. 1(c). Unexpectedly, the stability region of the bcc crystals covers a large area of the phase diagram, considerably more than in the monodisperse case. The first appearance of the bcc phase is always at a higher $\phi$ than that of fcc crystals, at the same $c_{s}$ (i.e., opposite to their order of occurrence in monodisperse CS systems [35]). Upon further compression, the system becomes a glass-forming liquid. To help explain these results, we use Monte Carlo (MC) simulations of our multicomponent model (MCM) for charge regulating polydisperse colloids parametrized with independent experimental data [19]. Allowing for only a slight adjustment of $\delta$, the simulations almost perfectly reproduce the experimental phase diagram.

For the experiments, we used industrially produced, nanometric and highly charged silica particles, dispersed in water (Ludox TM50, Sigma-Aldrich). These were cleaned and concentrated as detailed elsewhere [8,36-38]. Briefly, dispersions were filtered and dialyzed against aqueous $\mathrm{NaCl}$ solutions of various concentrations (from 0.5 to $50 \mathrm{mM}$ ) at $p \mathrm{H} 9 \pm 0.5$ (by addition of $\mathrm{NaOH}$ ). Next, they were slowly concentrated via the osmotic stress method, by the addition of polyethylene glycol (mw 35000, Sigma-Aldrich) outside the dialysis sack. Samples were then taken and sealed in quartz capillary tubes, on which small-angle $\mathrm{x}$-ray scattering (SAXS) experiments were performed at the ESRF, beamline ID02 [39]. The particle size distribution was measured in the dilute limit (see the Supplemental Material [18]) to have a mean size of $\bar{R}=13.75 \pm 1 \mathrm{~nm}$ and a polydispersity of $\delta=9 \pm 1 \%$, consistent with prior observations [20]. Over a range of concentrations the scattering spectra showed sharp peaks

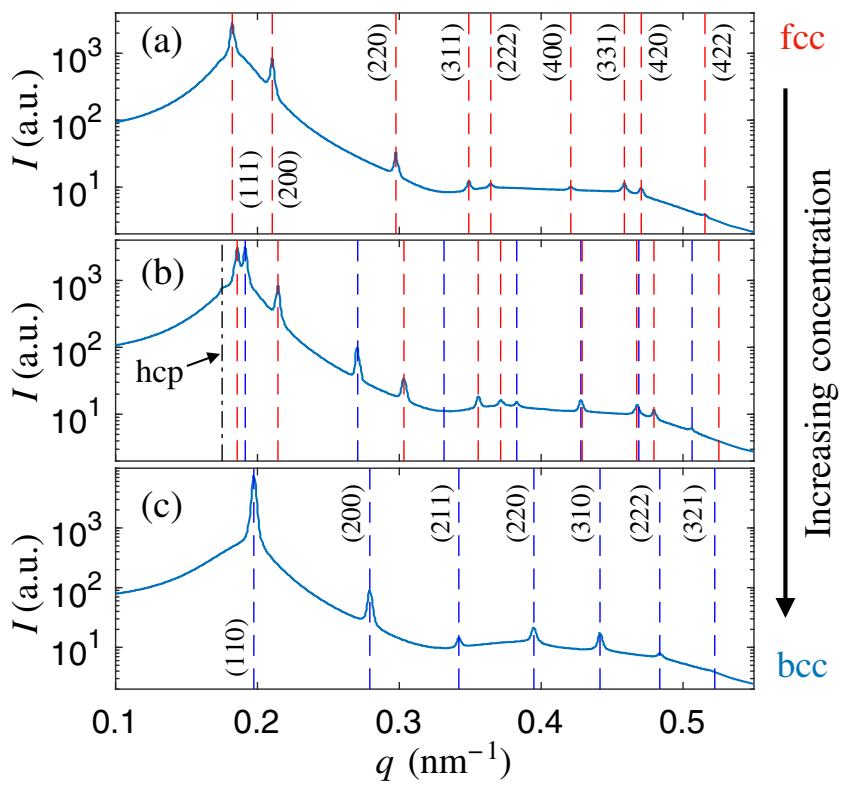

FIG. 2. As the dispersion is concentrated, colloidal crystals appear. The scattering intensities, $I$, of the spectra shown here, for $c_{s}=5 \mathrm{mM}$, demonstrate the typical sequence of (a) fcc $(\phi=19 \%)$, (b) a mixture of fcc and bcc $(\phi=20 \%)$, and (c) bcc $(\phi=21 \%)$ crystals, as $\phi$ increases. A broad liquid peak is present in all spectra, and the most prominent crystal peaks are typically at least twice as intense as this liquid background. Additionally, a much weaker peak is often visible at lower $q$, consistent with an hcp structure of the same particle density, or stacking faults in an fcc lattice.

characteristic of fcc and bcc crystal phases, as shown in Fig. 2. A weak peak representing a minority hop phase (or evidence of stacking faults [40]) was frequently seen alongside either crystal phase. Additional characterization of the liquid and glass phases is given in the Supplemental Material [18].

The experimental phase diagram in the $c_{s}-\phi$ plane is given in Fig. 3(a), and represents the phases that have nucleated and are experimentally stable over days to weeks. Whatever the background salinity, a fluid region is observed for low $\phi$ followed by a region with crystal formation at intermediate $\phi$, which ends in a re-entrant amorphous phase at high $\phi$. The latter behaves macroscopically as a solid (i.e., retains its shape as a soft gel or paste). As $c_{s}$ is increased, the first appearance of crystals shifts to higher $\phi$, in response to the screening of the electrostatic interactions. The same is true for the re-entrant melting transition. Both observations are consistent with phase diagrams of other experimental CS systems, although at much lower $c_{s}$ (e.g., $[41,42]$ ). The predominant ordered phases appearing are bcc and fcc crystals, also known from monodisperse CS systems (although the hep phase is not typically seen there, other than in shear-ordered samples [43]). However, the stability region of the bcc phase is observed at higher $\phi$ than the fcc phase, for all screening lengths studied (i.e., all $c_{s}$ ). This was also the case when we 
- Liquid $\Delta$ fcc $\square$ fcc + bcc $\nabla$ bcc $\circ$ Glass

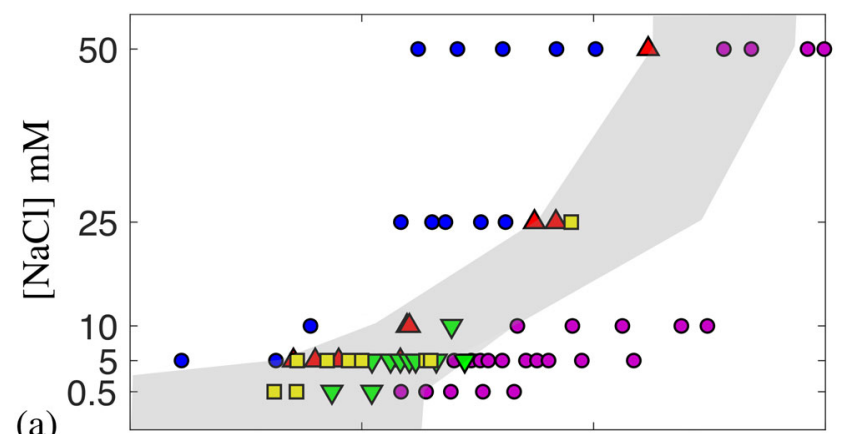

(a)

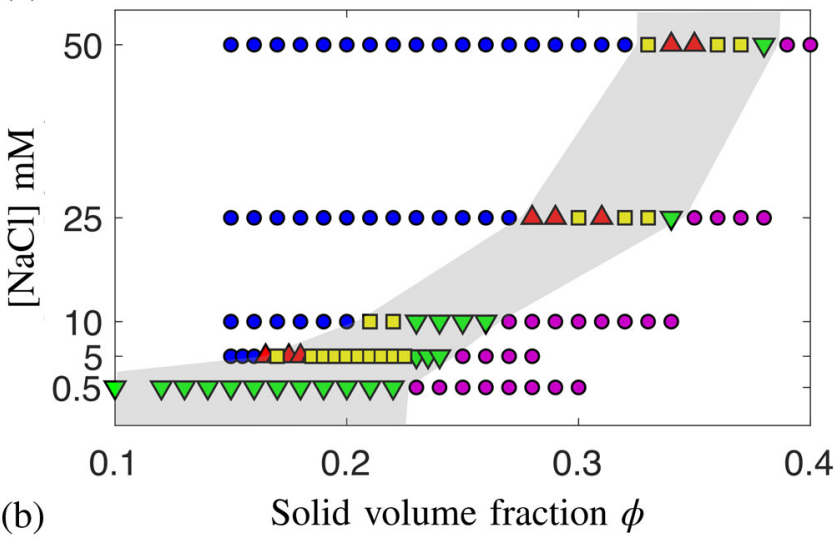

FIG. 3. Phase diagram in the $c_{s}-\phi$ plane of the TM50 silica dispersion at $p \mathrm{H} 9$ as obtained from (a) SAXS analysis of dialyzed samples and (b) MC simulations of the MCM. For clarity, the hcp phase is not represented. The shaded areas of (a), (b) delimit the region where crystals are found in the simulations, and demonstrate the good correspondence with experiments.

made a more continuous probe across $\phi$ as assessed by interdiffusion experiments (see the Supplemental Material [18] for details). This phase behavior contrasts strongly with that of monodisperse particles, where a bcc-fcc transition with increasing $\phi$ (rather than the fcc-bcc transition seen here) is invariably observed [41,42]. Essentially, this demonstrates that even a moderate polydispersity can have a complex influence on crystal stability, and modify the relative stability of various phases.

Although predicted to occur for soft colloids $[44,45]$ an inversion of the stability regions of the bcc and fcc phases has rarely been observed. To our knowledge, it has only been reported for soft spheres [46]. The possibility that polydispersity could help stabilize the bcc phase in CS systems was conjectured by some of us, based on an energetic argument which shows that the bcc structure is more tolerant to interaction polydispersity than the fcc one [13]. This argument was made via lattice MC simulations in the Gibbs ensemble on a system with a presupposed bccfcc coexistence of CS with $\delta=15 \%$. The particles were found to be divided up between a narrow monomodal distribution (fcc) and a bimodal one (bcc), similar to the situation given in Fig. 1(b). However, our reanalysis of this model shows that it also predicts the formation of a $\mathrm{CsCl}$ structure on the sites of the bcc phase (i.e., alternating larger and smaller particles), which is not compatible with our experimental findings (as additional scattering peaks would be present in this case).

Here we employed, instead, MC simulations for continuous systems at set density (NVT) or pressure (NPT) which do not require any prior information about the phases at equilibrium. They were performed at the experimental $c_{s}$ and $p \mathrm{H}$ conditions in the framework of the MCM detailed in Ref. [19], which includes the charge regulation of the silica particles through the $p \mathrm{H}$-dependent ionization of their surface active groups, $\mathrm{Si}-\mathrm{OH} \rightleftharpoons \mathrm{Si}-\mathrm{O}^{-}+\mathrm{H}^{+}$. A truncated and discretized Gaussian size distribution with the same $\bar{R}$ as measured was used, but with a somewhat lower polydispersity of $\delta=7 \%$ (rather than 9\%). Simple particle translations combined with swap moves [21] allow for efficiently sampling the phase space up to high $\phi$ [22]. Simulations were run with $N=19991$ particles in a cubic box with periodic boundary conditions. Up to several tens of million of MC cycles (each consisting of $N \mathrm{MC}$ moves) for equilibration were used; production runs lasted for $10^{5} \mathrm{MC}$ cycles. The local bond order parameters were used to analyze the obtained structures [23], and further details of the analysis and simulation are given in the Supplemental Material [18].

As shown in Fig. 3(b), a very good agreement is achieved between the experimental and simulated (MCM) phase diagrams. The same is true for the equation of state (EOS) of the TM50 silica dispersion in all the range of $c_{s}$ and $\phi$ studied as seen in Fig. 4(a) (experimental data from Refs. [38,47]). Not only is the inversion of the stability regions of the bcc and fcc phases well predicted, but also the position of the freezing transition matches with the experiments, although an exact phase diagram would require a free energy calculation not developed here (see the Supplemental Material [18] for discussion). In line with the experimental observations [38], a re-entrant amorphous phase at high $\phi$ is found, in which colloids present very weak diffusion.

The phase composition of the system upon compression at $c_{s}=5 \mathrm{mM}$ and an example of the size distributions at the coexistence of the hcp, bcc and fcc phases are shown in Figs. 4(b) and 4(c). The freezing transition is found to be first-order, and is characterized by both a discontinuity in the EOS and an abrupt change in the liquid and fcc phase composition at $\phi \approx 16 \%$, as in Fig. 4(b). The fcc-bcc phase transition is, on the other hand, found to be much more progressive. Simulation snapshots at the bcc-fcc-hcp phase coexistence show, instead, textures characteristic of a microphase separation (for further detail, see the Supplemental Material [18]).

The fcc-bcc phase transition is also characterized by a small size fractionation, as in Fig. 4(c), which tends to increase with $\phi$ (see the Supplemental Material [18]). The bcc phase is found to be more tolerant to 

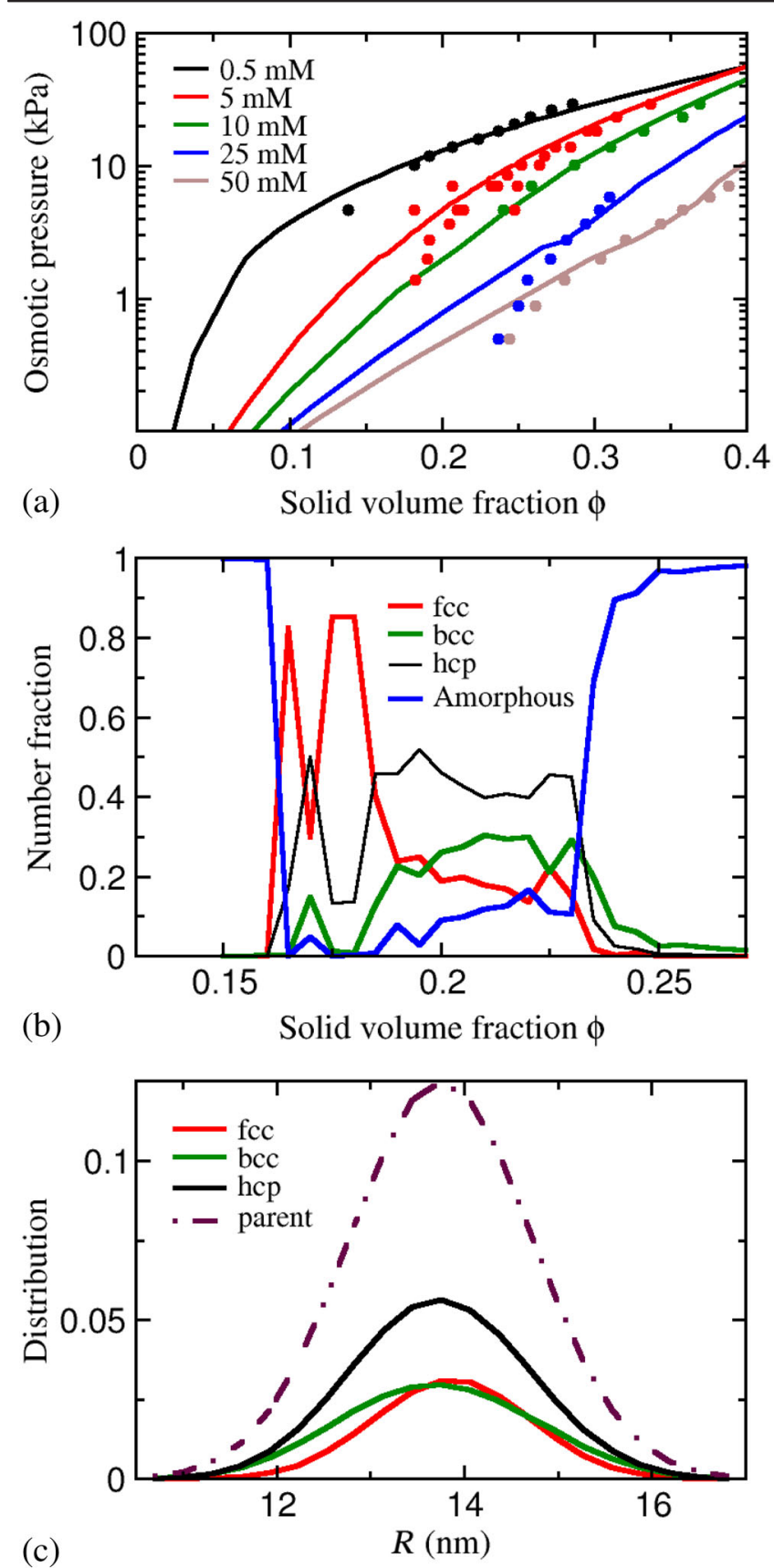

FIG. 4. Simulation results (at $p \mathrm{H}$ 9) show: (a) agreement of the simulated and measured equation of state (EOS) of the TM50 silica dispersion at various ionic strengths; (b) the predicted variation of the phase composition with $\phi$ at $c_{s}=5 \mathrm{mM}$; (c) and particle size distributions of the various crystalline phases in comparison with the parent size distribution (dashed curve) for the model silica dispersion at $\phi=20.5 \%, c_{s}=5 \mathrm{mM}$.

polydispersity, while incorporating a larger number of small particles than the fcc structure. The particle distribution of the bcc phase thus presents a larger $\delta$ and smaller $R$ than of the fcc phase. One consequence of this is that there will be only a small difference in the calculated particle number densities between both crystalline phases and the bulk, less than $4 \%$, with a slight tendency of the fcc phase to be the densest. This is consistent with our experimental observations, although the difference in phase densities falls within the uncertainty of the measurements (see the Supplemental Material [18]).

These results are in line with our energetic argument mentioned earlier [13]. In other words, the interaction polydispersity favors the formation of bcc crystals with a larger particle distribution (or charge distribution), thus being more tolerant to polydispersity, as compared to fcc crystals. As $\phi$ is progressively increased the fcc phase, compared to the bcc phase, becomes less and less tolerant to the charge polydispersity. Note that the latter is not constant but increases with $\phi$, see [19]. Consequently, the fcc ordered phase progressively disappears in favor of the bcc and fluid phases. Conversely, in the absence of interaction polydispersity the system can, to a good approximation, be reduced to that of point Yukawa particles [35]. In such a case, the charge of colloid $i$ satisfies the equality $Z_{i}^{*} \exp \left(-\kappa^{*} R_{i}\right) /\left(1+\kappa^{*} R_{i}\right)=\mathrm{C} \neq 0 \forall R_{i}$, where $C$ is a constant (see the Supplemental Material [18]). The inversion of the stability regions of the bcc and fcc phases is then lost [48]. In this case the stability region of the bcc is also restricted to the very diluted $c_{s}-\phi$ domain only. In absence of charge (i.e., $C=0$ ), the bcc phase simply disappears, see, e.g., the recent work of Bommineni on polydisperse HS systems [9]. All this further illustrates the importance of charge dispersity in the inversion of the stability regions of the bcc and fcc phases.

Obviously, the phase behavior observed in our experiments and simulations is not only a consequence of the system's internal energy, but the result of the balance between energy and entropy. In an attempt to elucidate the entropic contributions in the stabilization of the bcc phase we further performed lattice simulations in the Gibbs ensemble, as in Ref. [13], with the MCM of the TM50 silica dispersion. As in the continuous simulations, a small size fractionation is obtained. However, a $\mathrm{CsCl}$ superlattice structure, instead of a bcc phase, is found (see the Supplemental Material [18]). Recognizing that lattice simulations only account for the mixing contribution to the entropy, one can deduce from this qualitative difference that the bcc phase observed in our experiments (and continuous simulations) is most probably stabilized by vibrational entropy (the missing thermodynamic ingredient in lattice simulations). A large size fractionation in distinct phases is, on the other hand, prevented by the mixing entropy at this relatively small $\delta$ and range of $\phi$. When the size polydispersity is increased (see the Supplemental Material [18]), the mixing entropy takes over, and a $\mathrm{MgZn}_{2}$ Laves phase in coexistence with a bcc phase is predicted to occur in good agreement with our previous experimental findings [8].

Not discussed so far is the striking agreement obtained between the simulations and experiments on the position of 
the re-entrant melting line. At a first sight, this would suggest that the amorphous phase is stable. Preliminary results obtained well inside the amorphous region with more advanced simulation techniques show, however, that it can crystallize. A close look at the EOS also shows a sudden increase in the osmotic pressure. These results, which will be developed elsewhere, strongly suggest that it is a glass forming liquid. Still, we were unable to come with a reasonable explanation for the troubling coincidence between our simulation and experimental results on the (nonthermodynamic) re-entrant melting transition.

To conclude, using a combined and detailed theoretical and experimental study of charged nanocolloids with a moderate polydispersity, we provide evidence that the packing of polydisperse particles into crystals is much more diverse than initially thought, even for relatively small polydispersities. In particular, the system is found to separate into coexisting solid phases with a limited size fractionation. Under compression, the system first solidifies in compact lattice structures, fcc and hcp. Upon further compression, the fcc phase dissolves progressively into a less compact bcc structure, which proves to be more tolerant to the interaction (charge) polydispersity. Our simulations strongly suggest that the limited size fractionation and the stabilization of the bcc phase are due to the mixing and vibrational entropies, respectively. Compressed even further, the colloidal crystals melt into an amorphous phase, most probably a glass forming liquid. The astonishingly good agreement obtained between our experimental results and simulated predictions further gives a strong support to the simulation methods employed and the parameter-free force field developed. We anticipate that these tools should help in the finding of new colloidal crystal phases and in providing a better understanding of colloidal glasses in CS systems. Still, the exact phase boundaries and equilibrium phase behavior of polydisperse $\mathrm{CS}$, in particular at high densities, remain open questions which will require the development of advanced simulation techniques to be tackled.

We acknowledge the European Synchrotron Radiation Facility for provision of synchrotron radiation facilities and assistance in using beam line ID02. G. B. and C. L. thanks financial support from the Region Bourgogne FrancheComté and CNRS, as well as computational support from CRI, Université de Bourgogne. P.-C. K. thanks the Thai DPST and the Royal Government of Thailand for funding.

G. B. and P.-C. K. contributed equally to this work.

*lucas.goehring@ntu.ac.uk

Christophe.labbez@u-bourgogne.fr

[1] P. N. Pusey, The effect of polydispersity on the crystallization of hard spherical colloids, J. Phys. II (France) 48, 709 (1987).
[2] P. N. Pusey, Colloidal suspensions, in Les Houches Session L1: Liquids, Freezing, and the Glass Transition, edited by J. P. Hansen, D. Levesque, and J. Zinn-Justin (North-Holland, Les Houche, 1991), pp. 765-942.

[3] S. Auer and D. Frenkel, Suppression of crystal nucleation in polydisperse colloids due to increase of the surface free energy, Nature (London) 413, 711 (2001).

[4] P. N. Pusey, E. Zaccarelli, C. Valeriani, E. Sanz, W. C. K. Poon, and M. E. Cates, Hard spheres: Crystallization and glass formation, Philos. Trans. R. Soc. Lond. Math. Phys. Eng. Sci. 367, 4993 (2009).

[5] D. A. Kofke and P. G. Bolhuis, Freezing of polydisperse hard spheres, Phys. Rev. E 59, 618 (1999).

[6] M. Fasolo and P. Sollich, Equilibrium Phase Behavior of Polydisperse Hard Spheres, Phys. Rev. Lett. 91, 068301 (2003).

[7] P. Sollich and N. B. Wilding, Crystalline Phases of Polydisperse Spheres, Phys. Rev. Lett. 104, 118302 (2010).

[8] B. Cabane, J. Li, F. Artzner, R. Botet, C. Labbez, G. Bareigts, M. Sztucki, and L. Goehring, Hiding in Plain View: Colloidal Self-Assembly from Polydisperse Populations, Phys. Rev. Lett. 116, 208001 (2016).

[9] P. K. Bommineni, N. R. Varela-Rosales, M. Klement, and M. Engel, Complex Crystals from Size-Disperse Spheres, Phys. Rev. Lett. 122, 128005 (2019).

[10] E. V. Shevchenko, D. V. Talapin, N. A. Kotov, S. O'Brien, and C. B. Murray, Structural diversity in binary nanoparticle superlattices, Nature (London) 439, 55 (2006).

[11] A.-P. Hynninen, J. H. J. Thijssen, E. C. M. Vermolen, M. Dijkstra, and A. van Blaaderen, Self-assembly route for photonic crystals with a bandgap in the visible region, Nat. Mater. 6, 202 (2007).

[12] N. Schaertl, D. Botin, T. Palberg, and E. Bartsch, Formation of Laves phases in buoyancy matched hard sphere suspensions, Soft Matter 14, 5130 (2018).

[13] R. Botet, B. Cabane, L. Goehring, J. Li, and F. Artzner, How do polydisperse repulsive colloids crystallize?, Faraday Discuss. 186, 229 (2016).

[14] D. Coslovich, M. Ozawa, and L. Berthier, Local order and crystallization of dense polydisperse hard spheres, J. Phys. Condens. Matter 30, 144004 (2018).

[15] B. A. Lindquist, R. B. Jadrich, and T. M. Truskett, Communication: From close-packed to topologically closepacked: Formation of Laves phases in moderately polydisperse hard-sphere mixtures, J. Chem. Phys. 148, 191101 (2018).

[16] L. A. Fernández, V. Martín-Mayor, and P. Verrocchio, Phase Diagram of a Polydisperse Soft-Spheres Model for Liquids and Colloids, Phys. Rev. Lett. 98, 085702 (2007).

[17] D. V. Byelov, M. C. D. Mourad, I. Snigireva, A. Snigirev, A. V. Petukhov, and H. N. W. Lekkerkerker, Experimental observation of fractionated crystallization in polydisperse platelike colloids, Langmuir 26, 6898 (2010).

[18] See the Supplemental Material at http://link.aps.org/ supplemental/10.1103/PhysRevLett.124.058003 for an extended materials and methods section, as well as for details of the suspension size distribution, interdiffusion experiments, phase composition, glassy state, simulation convergence, and lattice simulations, which includes Refs. [8,13,14,19-34]. 
[19] G. Bareigts and C. Labbez, Jellium and cell model for titratable colloids with continuous size distribution, J. Chem. Phys. 149, 244903 (2018).

[20] V. Goertz, N. Dingenouts, and H. Nirschl, Comparison of nanometric particle size distributions as determined by SAXS, TEM and analytical ultracentrifuge, Part. Part. Syst. Charact. 26, 17 (2009).

[21] T. S. Grigera and G. Parisi, Fast Monte Carlo algorithm for supercooled soft spheres, Phys. Rev. E 63, 045102(R) (2001).

[22] C. Brito, E. Lerner, and M. Wyart, Theory for Swap Acceleration near the Glass and Jamming Transitions for Continuously Polydisperse Particles, Phys. Rev. X 8, 031050 (2018).

[23] W. Lechner and C. Dellago, Accurate determination of crystal structures based on averaged local bond order parameters, J. Chem. Phys. 129, 114707 (2008).

[24] R. Botet and B. Cabane, Simple inversion formula for the small-angle X-ray scattering intensity from polydisperse systems of spheres, J. Appl. Crystallogr. 45, 406 (2012).

[25] J. Li, B. Cabane, M. Sztucki, J. Gummel, and L. Goehring, Drying dip-coated colloidal films, Langmuir 28, 200 (2012).

[26] N. Boon, G. I. Guerrero-García, R. van Roij, and M. O. de la Cruz, Effective charges and virial pressure of concentrated macroion solutions, Proc. Natl. Acad. Sci. U.S.A. 112, 9242 (2015).

[27] G. H. Bolt, Determination of the charge density of silica sols, J. Phys. Chem. 61, 1166 (1957).

[28] C. Labbez, B. Jonsson, M. Skarba, and M. Borkovec, Ion-ion correlation and charge reversal at titrating solid interfaces, Langmuir 25, 7209 (2009).

[29] P. M. Dove and C. M. Craven, Surface charge density on silica in alkali and alkaline earth chloride electrolyte solutions, Geochim. Cosmochim. Acta 69, 4963 (2005).

[30] D. Frenkel and B. Smit, Understanding Molecular Simulation (Academic Press, San Diego, 2002).

[31] N. B. Wilding and P. Sollich, Phase behavior of polydisperse spheres: Simulation strategies and an application to the freezing transition, J. Chem. Phys. 133, 224102 (2010).

[32] L. Verlet, Computer "Experiments" on classical fluids. II. Equilibrium correlation functions, Phys. Rev. 165, 201 (1968).

[33] J.-P. Hansen and L. Verlet, Phase transitions of the LennardJones system, Phys. Rev. 184, 151 (1969).

[34] M. Leocmach, J. Russo, and H. Tanaka, Importance of many-body correlations in glass transition: An example from polydisperse hard spheres, J. Chem. Phys. 138, 12A536 (2013).
[35] A.-P. Hynninen and M. Dijkstra, Phase diagrams of hardcore repulsive Yukawa particles, Phys. Rev. E 68, 021407 (2003).

[36] B. Jönsson, J. Persello, J. Li, and B. Cabane, Equation of state of colloidal dispersions, Langmuir 27, 6606 (2011).

[37] J. Li, M. Turesson, C. A. Haglund, B. Cabane, and M. Skepö, Equation of state of PEG/PEO in good solvent. Comparison between a one-parameter EOS and experiments, Polymer 80, 205 (2015).

[38] L. Goehring, J. Li, and P.-C. Kiatkirakajorn, Drying paint: From micro-scale dynamics to mechanical instabilities, Phil. Trans. R. Soc. A 375, 20160161 (2017).

[39] T. Narayanan, M. Sztucki, P. Van Vaerenbergh, J. Léonardon, J. Gorini, L. Claustre, F. Sever, J. Morse, and P. Boesecke, A multipurpose instrument for time-resolved ultra-small-angle and coherent X-ray scattering, J. Appl. Crystallogr. 51, 1511 (2018).

[40] A. G. Shabalin, J.-M. Meijer, R. Dronyak, O. M. Yefanov, A. Singer, R. P. Kurta, U. Lorenz, O. Y. Gorobtsov, D. Dzhigaev, S. Kalbfleisch, J. Gulden, A. V. Zozulya, M. Sprung, A. V. Petukhov, and I. A. Vartanyants, Revealing Three-Dimensional Structure of an Individual Colloidal Crystal Grain by Coherent X-Ray Diffractive Imaging, Phys. Rev. Lett. 117, 138002 (2016).

[41] Y. Monovoukas and A. P. Gast, The experimental phase diagram of charged colloidal suspensions, J. Colloid Interface Sci. 128, 533 (1989).

[42] E. B. Sirota, H. D. Ou-Yang, S. K. Sinha, P. M. Chaikin, J.D. Axe, and Y. Fujii, Complete Phase Diagram of a Charged Colloidal System: A Synchrotron X-Ray Scattering Study, Phys. Rev. Lett. 62, 1524 (1989).

[43] H. Versmold, Neutron Diffraction from Shear Ordered Colloidal Dispersions, Phys. Rev. Lett. 75, 763 (1995).

[44] D. Gottwald, C. N. Likos, G. Kahl, and H. Löwen, Phase Behavior of Ionic Microgels, Phys. Rev. Lett. 92, 068301 (2004).

[45] J. C. Pàmies, A. Cacciuto, and D. Frenkel, Phase diagram of Hertzian spheres, J. Chem. Phys. 131, 044514 (2009).

[46] P. S. Mohanty and W. Richtering, Structural ordering and phase behavior of charged microgels, J. Phys. Chem. B 112, 14692 (2008).

[47] P.-C. Kiatkirakajorn, Morphological instabilities in drying colloids, Ph. D. thesis, Georg-August University, Göttingen, 2018.

[48] S. Hamaguchi, R. T. Farouki, and D. H. E. Dubin, Triple point of Yukawa systems, Phys. Rev. E 56, 4671 (1997). 